\title{
Searches for supersymmetry via strong production in events with one or more leptons at CMS
}

\author{
Christian Schomakers* \\ On behalf of the CMS Collaboration \\ I. Physikalisches Institut B, RWTH Aachen University (DE) \\ E-mail: christian.schomakers@cern.ch
}

Five searches for new physics are presented in events with one or more charged light leptons, jets and missing transverse momentum. The searches use $35.9 \mathrm{fb}^{-1}$ of $13 \mathrm{TeV}$ proton-proton collision data recorded in 2016 by the CMS experiment at the LHC. No significant excess with respect to the background estimation is observed and the results are interpreted in terms of several simplified models of pair production of supersymmetric partners of gluons or quarks. Depending on the model, gluino masses up to $1.9 \mathrm{TeV}$ and squark masses up to $1.2 \mathrm{TeV}$ are excluded.

The European Physical Society Conference on High Energy Physics

5-12 July

Venice, Italy

\footnotetext{
* Speaker.
} 


\section{Introduction}

Five searches for supersymmetry (SUSY), produced via the strong interaction, in final states with at least one lepton, jets, and missing transverse momentum $\left(p_{\mathrm{T}}^{\text {miss }}\right)$ are presented. The results are based on $35.9 \mathrm{fb}^{-1}$ of proton-proton collision data recorded at a center-of-mass energy of $13 \mathrm{TeV}$ with the CMS experiment [1] in 2016.

For a hadron collider, like the LHC, the production of new particles via the strong interaction is assumed to have the largest cross section in most SUSY scenarios. Many SUSY models that are considered "natural" feature light top squarks. Assuming flavor and R-parity conservation, the decays of these squarks yield top quarks and $p_{\mathrm{T}}^{\text {miss }}$ from the lightest supersymmetric particle (LSP), which is assumed to be the lightest neutralino $\tilde{\chi}_{1}^{0}$ in this report and escapes undetected. Similar final states can originate from the pair production of gluinos or bottom squarks.

While the decay into a fully hadronic final state has the largest branching fraction for most such models, it also suffers from a large background contribution from standard model (SM) events containing uniquely jets produced through the strong interaction (QCD multijet background). Requiring one or more leptons, e.g. from the decays of top quarks, reduces the branching fraction of SUSY models but also suppresses the QCD multijet background contribution significantly. Correlated lepton pairs can arise from decay chains resulting in $\mathrm{Z}$ bosons or including intermediate sleptons. Examples for a few of the considered simplified SUSY models are shown in Fig. 1.

An overview of the various searches is given in the following sections. More details, especially additional interpretations, e. g. in electroweakly produced SUSY models, can be found in the main documentation of the results. Since electrons and muons are easier to distinguish from jets than tau leptons, only light leptons are considered in these analyses.
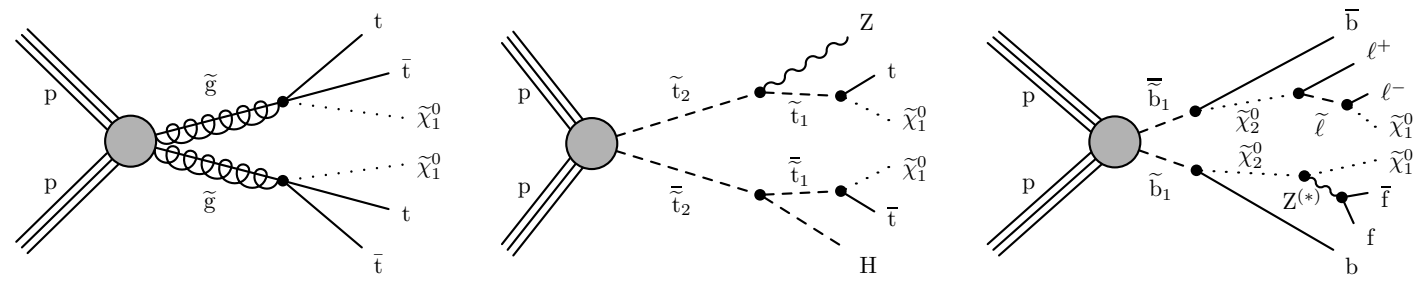

Figure 1: Graphs for some of the considered simplified SUSY models. Left: Pair production of gluinos, each decaying into two top quarks and the LSP. Center: Pair production of the heavier top squark, decaying into the lighter top squark and either a Higgs or Z boson. The lighter top squark decays into a top quark and the LSP. Right: Pair production of bottom squarks, decaying into a b quark and a $\tilde{\chi}_{2}^{0}$, which further decays into the LSP either via an intermediate slepton or the emission of a $\mathrm{Z}$ boson.

\section{Single lepton search with large jets}

The first search targets the pair production of gluinos, decaying into top quark pairs and the LSP [2]. Such a final state features a high number of jets, several of them b-tagged, a significant amount of $p_{\mathrm{T}}^{\text {miss }}$, and a large probability to produce at least one light lepton. Therefore, the basic event selection requires exactly one lepton, at least six jets, at least one of those b-tagged, and more 
than $200 \mathrm{GeV}$ of $p_{\mathrm{T}}^{\text {miss }}$. Furthermore, the sum of the $p_{\mathrm{T}}$ of the lepton and the hadronic activity $\left(H_{\mathrm{T}}\right)$, defined as the scalar sum of all transverse jet momenta, is required to be larger than $500 \mathrm{GeV}$.

The main background contribution in this selection arises from top quark pairs (t⿱tt) decaying semileptonically and can be reduced by a requirement on the transverse mass $\left(m_{\mathrm{T}}=\sqrt{2 p_{\mathrm{T}}^{\ell}\left[1-\cos \left(\Delta \phi_{\ell, \vec{p}_{\mathrm{T}}^{\text {miss }}}\right)\right]}\right)$. An additional uncorrelated observable $M_{J}$ is obtained by reclustering all jets and leptons using an anti- $k_{\mathrm{T}}$ algorithm [3] with a distance parameter of 1.4 and summing up masses of the resulting jets. In case of $t \bar{t}$, a cutoff of the $M_{J}$ distribution at two times the top mass is expected. The presence of jets from initial state radiation generates a contribution of the $t \bar{t}$ background at high values of $M_{J}$.

Events are required to fulfill $m_{\mathrm{T}}>140 \mathrm{GeV}$ and $M_{J}>400 \mathrm{GeV}$ to enter the signal selection and signal bins are defined in $p_{\mathrm{T}}^{\text {miss }}$, jet, and $\mathrm{b}$ jet multiplicity. Three control regions, $\mathrm{R} 1-3$, are defined at low values of $m_{\mathrm{T}}$ or $M_{J}$, as indicated in the left plot in Fig. 2. A fit to the three control regions provides the background prediction and a global fit on all regions is used for the interpretation in signal models. The residual $m_{\mathrm{T}}-M_{J}$ correlations, obtained from simulation, are used to constrain the fit.

The observation agrees with the expectation from SM processes within 2 standard deviations (s. d.) in all signal regions and the results are interpreted in two simplified model based on gluino pair production as shown in the right plot of Fig. 2. The gluino decays either directly or via an intermediate top squark into two top quarks and the LSP. Very similar exclusion limits are obtained for both models at 95\% confidence level (CL). An exception are weaker limits for the model with intermediate top squarks at low LSP masses. In both scenarios, gluino masses up to $1.9 \mathrm{TeV}$ are excluded, depending on the LSP mass.
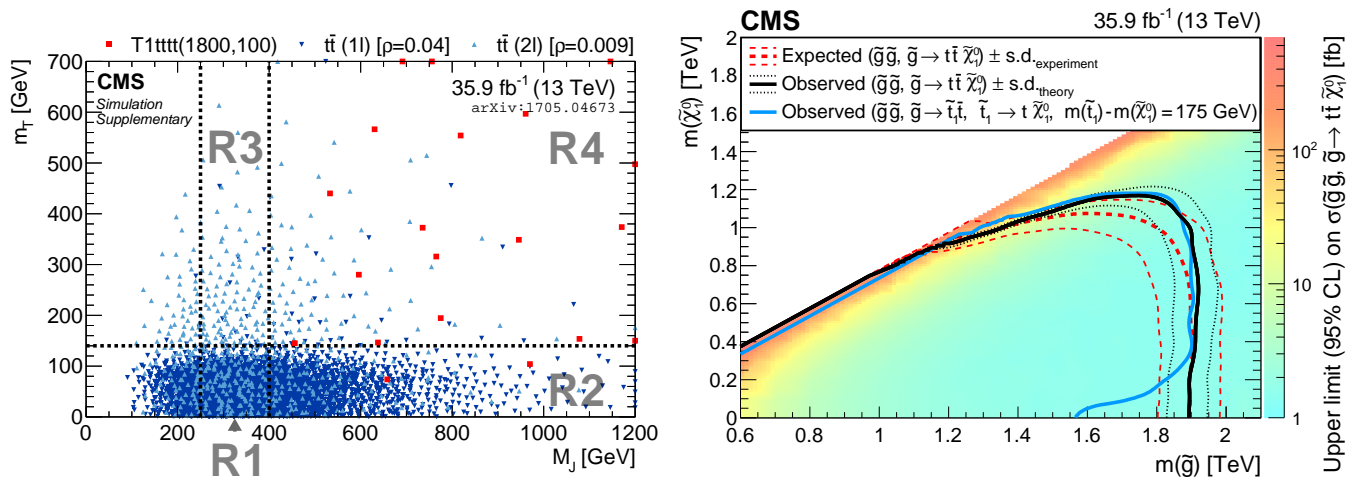

Figure 2: Left: Simulated single-lepton $\mathrm{t} \overline{\mathrm{t}}$ (dark-blue triangles), dilepton $\mathrm{t} \overline{\mathrm{t}}$ (light-blue triangles), and example signal events (red squares) in the $M_{J}-m_{\mathrm{T}}$ plane after the baseline selection of the single lepton search with large jets [2]. Each marker represents one expected event at $35.9 \mathrm{fb}^{-1}$. The values of the correlation coefficients for each background process are given as well. Right: Interpretation of the results in models for gluino pair production [2]. The color coding shows the observed cross section upper limits at 95\% CL for direct decays of the gluino into a top quark pair and the LSP. The expected and observed exclusion contours in the same model are indicated by dashed red and solid black lines, respectively. The observed mass limit for a model with intermediate top squark is shown as a solid blue line. 


\section{Single lepton search with $\Delta \phi$}

The second analysis searches for SUSY in events with a single lepton as well, but targets final states that do not necessarily include top quarks [4]. The signal region requires the scalar sum of the lepton $p_{\mathrm{T}}$ and $p_{\mathrm{T}}^{\text {miss }}$, referred to as $L_{\mathrm{T}}$, to be larger than $250 \mathrm{GeV}$, at least five jets, and $H_{\mathrm{T}}>500 \mathrm{GeV}$. No general requirement on the number of b-tagged jets is made, but the signal region is split into events with and without b-tagged jets. The former selection is dominated by semileptonic $t \bar{t}$ decays, while the latter contains similar fractions of events from $\mathrm{W}+j$ jets and $t \bar{t}$. In both processes, the lepton originates from the decay of a $\mathrm{W}$ boson and a small azimuthal angle $\Delta \phi$ is expected between the lepton and the $\mathrm{W}$ boson candidate reconstructed from the lepton and the missing transverse momentum vector $\left(\vec{p}_{\mathrm{T}}^{\mathrm{miss}}\right)$. Since no such correlation is expected for the considered SUSY scenarios, the signal regions are defined at large $\Delta \phi$ with thresholds between 0.5 and 1 , depending on the signal bin.

Control regions at low $\Delta \phi$ are used to predict the background, using additional control regions at low jet multiplicity to obtain transfer factors from low to high values of $\Delta \phi$. As in Section 2, residual corrections are obtained from simulation. Additionally, a small contribution of QCD multijet events that only contributes at low $\Delta \phi$ is determined using a tight-to-loose ratio method and is subtracted from the yield in the control region before applying the transfer factor.

The signal regions with and without b-tagged jet are further split into bins of jet multiplicity, $H_{\mathrm{T}}, L_{\mathrm{T}}$, and, in case of the b-tagged region, b jet multiplicity. The observed and predicted yields are compared in Fig. 3. The observed data are found to agree with the background prediction and limits on simplified models of gluino pair production up to $1.9 \mathrm{TeV}$ are set.
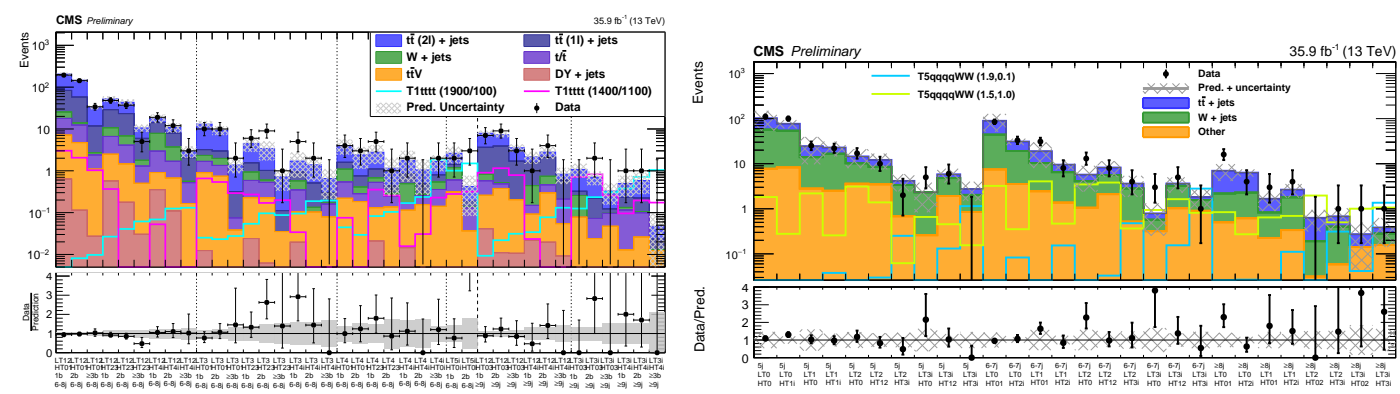

Figure 3: Comparison of the predicted and observed yields in the signal regions of the single lepton search with $\Delta \phi$ with (left) and without b-tagged jets (right) [4]. The relative fraction of the different SM background contributions, obtained from simulation, is shown as a stacked histogram normalized to the background estimation from data control regions. The expected yields for two example points of different models based on gluino pair production are displayed as well. The lower panel shows the ratio of observed events in data to the prediction. The error bars on the data points indicate the combined statistical and systematic uncertainty. The hatched bands in both panels display the uncertainty on the background prediction.

\section{Opposite-sign, same-flavor dilepton search}

Requiring a second lepton reduces the QCD multijet background contribution to a negligible fraction and suppresses events from $\mathrm{W}+\mathrm{jets}$ and semileptonic $\mathrm{t} \overline{\mathrm{t}}$ decays. This allows the oppositesign, same-flavor (OSSF) dilepton search to reduce the requirement on the jet multiplicity to at 
least two jets [5]. The dominant background from Drell-Yan (DY) processes is suppressed by requiring more than 100 to $150 \mathrm{GeV}$ of $p_{\mathrm{T}}^{\text {miss }}$, depending on the signal region, and a difference in the azimuthal angle between each of the two leading jets and $\vec{p}_{\mathrm{T}}^{\text {miss }}$ of more than 0.4. Dileptonic $t \bar{t}$ decays are the dominant remaining background process. The number of background events from this process is reduced by a requirement on the $M_{\mathrm{T} 2}$ variable [6] of $80 \mathrm{GeV}$.

Three background contributions remain: flavor-symmetric (FS) processes, which produce opposite-flavor (OF) pairs as often as same-flavor (SF) pairs, DY+jets events without neutrinos from the hard interaction, and rare SM processes with a $\mathrm{Z}$ boson and neutrinos. Yields in a control sample of electron-muon events, corrected for differences in the lepton efficiencies, are used to predict the FS background. In DY+jets events, the $p_{\mathrm{T}}^{\text {miss }}$ arises mainly from mismeasurements of the hadronic system. The same is true for $\gamma+$ jets events and photon data are used to predict the $p_{\mathrm{T}}^{\text {miss }}$ spectrum in DY+jets events using the so-called $p_{\mathrm{T}}^{\text {miss }}$-templates method. The contribution from rare $\mathrm{SM}$ processes is taken from simulation and validated in multilepton control regions.

The OSSF dilepton search targets two signatures: a resonant contribution on the $\mathrm{Z}$ boson mass peak (on- $Z$ search) and a kinematic edge in the invariant dilepton mass $\left(m_{\ell \ell}\right)$ spectrum outside of the $\mathrm{Z}$ boson mass window (edge search). The former can occur in SUSY processes if an on-shell $\mathrm{Z}$ boson is produced, while the latter arises from off-shell $\mathrm{Z}$ bosons or decays via intermediate sleptons. The on-Z search features several bins in the multiplicity of (b-tagged) jets and $p_{\mathrm{T}}^{\text {miss }}$, while the edge search performs a counting experiment in bins of $m_{\ell \ell}$ and a t⿱t-t- likelihood discriminator as well as a kinematic fit in the full mass range.

Figure 4 shows the results in the different signal bins and the results of the kinematic fit. The largest deviation from the expectation of about $2 \mathrm{~s} . \mathrm{d}$. is observed in the not-tit-like mass bin from 96 to $150 \mathrm{GeV}$ of the edge search. The fit to search for a kinematic edge results in $61 \pm 28$ signal events for an edge position of about $145 \mathrm{GeV}$, which is compatible with the results of the counting experiment. Taking the look-elsewhere-effect introduced by the floating edge position into account, a significance of $1.5 \mathrm{~s}$. d. is obtained. The results of the on- $Z$ search are used to set limits of up to $1.8 \mathrm{TeV}$ on gluino masses in a simplified model based on gauge-mediated supersymmetry breaking, while the edge search probes bottom quark masses up to $1.2 \mathrm{TeV}$ in the model with an intermediate slepton shown in the right plot of Fig. 1.
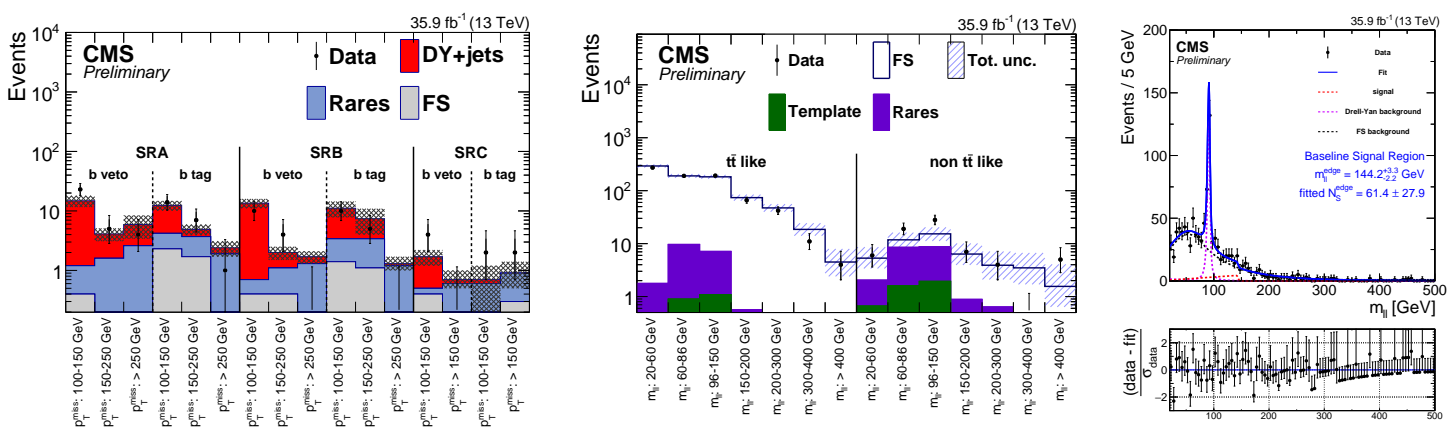

Figure 4: Results of the OSSF dilepton search [5]. The observation in the signal regions to search for a resonant contribution at the $\mathrm{Z}$ boson mass (left) and for a deviation outside the $\mathrm{Z}$ boson mass window (center) are compared to the background prediction. The best fit to search for a kinematic edge is shown as well (right). 


\section{Same sign dilepton and multilepton search}

Final states with a same sign lepton pair or more than two leptons are very rare in the SM. This allows the two searches to consider events with at least two jets and more than $50 \mathrm{GeV}$ of $p_{\mathrm{T}}^{\text {miss }}$ in their signal selections [7, 8]. In case of the same sign search, events with an additional loose lepton are vetoed if this lepton can be combined with one of the two main leptons to form an OSSF pair that is compatible with the $\mathrm{Z}$ boson mass or a low mass resonance.

The main background contribution in the same sign search and for multilepton events without $\mathrm{Z}$ boson candidate originates from events with at least one nonprompt lepton, i.e. a jet that is misidentified as a lepton or a lepton from a heavy-flavor quark decay. Rare SM processes which produce a same sign pair or at least three leptons are estimated from simulation. In both analyses, the most important production processes are $\mathrm{WZ}$ and $\mathrm{t} \mathrm{tZ}$ production. Data control regions are defined to validate the simulation of these processes and obtain corrections for the overall normalization. In the same sign search, a minor background contribution arises from charge-misidentified electrons. The misidentification rate is determined on simulation, validated in data control regions, and applied to opposite-sign dilepton data.

The same sign search splits the signal region into bins according to the lepton momenta and charges, while the multilepton search divides the selection into events with and without a $\mathrm{Z}$ boson candidate. Both searches use additional signal bins in the jet and $\mathrm{b}$ jet multiplicity, $H_{\mathrm{T}}, p_{\mathrm{T}}^{\text {miss }}$, and $m_{\mathrm{T}}$. If a $\mathrm{Z}$ boson candidate is present, the remaining lepton is combined with $\vec{p}_{\mathrm{T}}^{\mathrm{miss}}$ to calculate $m_{\mathrm{T}}$, otherwise all combinations of a lepton with $\vec{p}_{\mathrm{T}}^{\text {miss }}$ are tested and the minimal value is used.

The results of both searches are summarized in Fig. 5. No significant deviation from the expectation is observed and limits on a variety of simplified models are set with upper limits on the top and bottom squark masses of up to $850-900 \mathrm{GeV}$ and gluino masses up to $1.6 \mathrm{TeV}$, depending on the exact model.

\section{Conclusion}

Results of five searches for strongly produced supersymmetry in events with at least one light lepton have been presented. The data were recorded by the CMS experiment at a center-of-mass energy of $13 \mathrm{TeV}$ in 2016. No significant deviation from the standard model expectation is observed and the results are interpreted in various simplified models. Gluino masses up to $1.9 \mathrm{TeV}$, top squark masses up to $900 \mathrm{GeV}$, and bottom squark masses up to $1.2 \mathrm{TeV}$ are probed.

\section{References}

[1] CMS Collaboration, "The CMS experiment at the CERN LHC", JINST 3 (2008) S08004, doi: $10.1088 / 1748-0221 / 3 / 08 / \mathrm{s} 08004$

[2] CMS Collaboration, "Search for supersymmetry in pp collisions at $\sqrt{s}=13 \mathrm{TeV}$ in the single-lepton final state using the sum of masses of large-radius jets", Accepted by Phys. Rev. Lett., arXiv: 1705.04673

[3] M. Cacciari, G. P. Salam, and G. Soyez, "The anti-kt jet clustering algorithm", JHEP 04 (2008) 063, doi:10.1088/1126-6708/2008/04/063, arXiv:0802.1189 

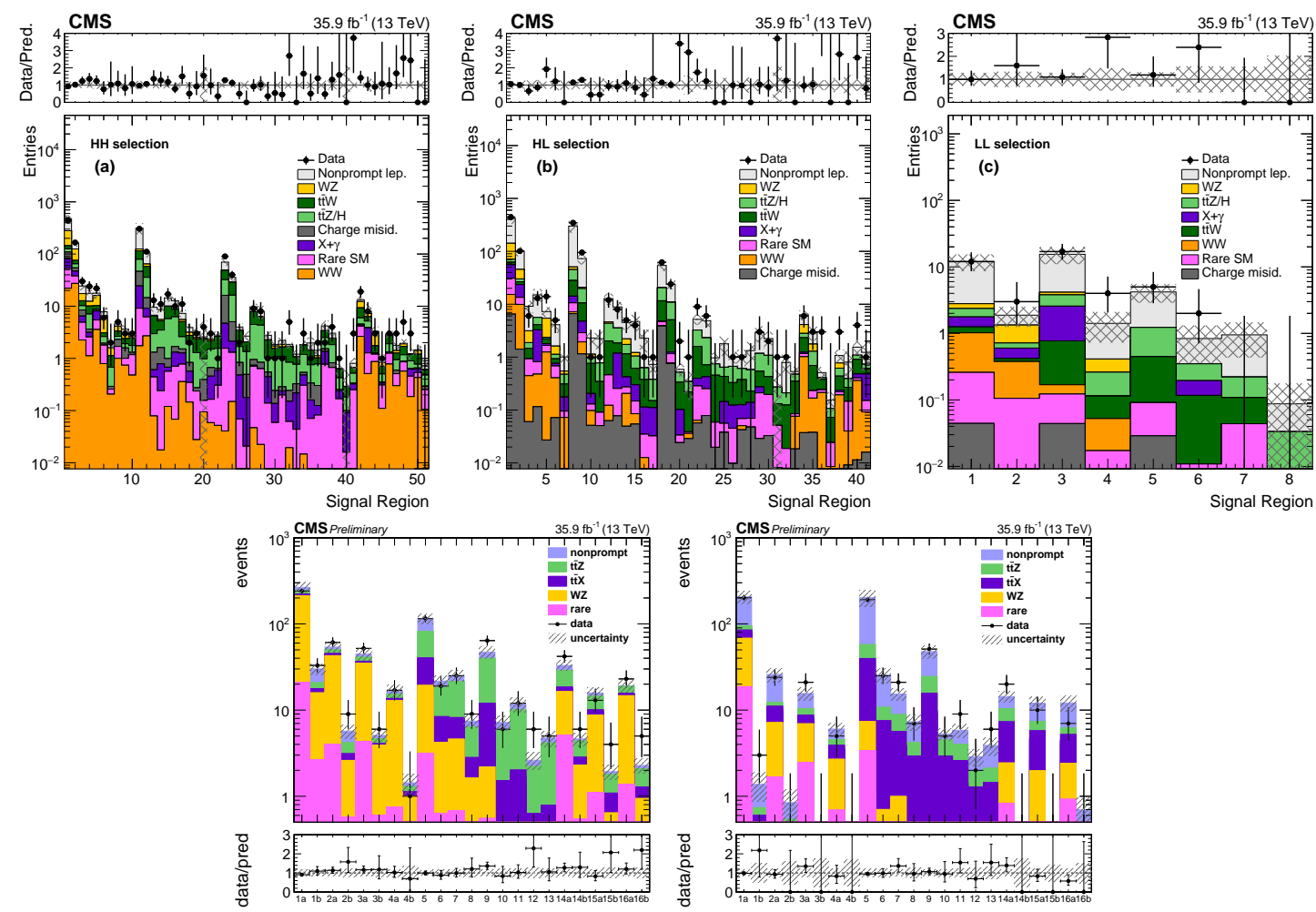

Figure 5: Results of the same sign (top) [7] and multilepton searches (bottom) [8]. The same sign results are split into events where both leptons have $p_{\mathrm{T}}>25 \mathrm{GeV}$ (left) and those with one (center) or both leptons reconstructed with $10<p_{\mathrm{T}}<25 \mathrm{GeV}$ (right). The results of the multilepton search are split into events with (left) and without a $\mathrm{Z}$ boson candidate (right). The background contributions are stacked and compared to the observed data in the main panel, while the ratio of data yield to background prediction is displayed in the smaller upper or lower panel.

[4] CMS Collaboration, "Search for supersymmetry in events with one lepton and multiple jets in proton-proton collisions at $\sqrt{s}=13 \mathrm{TeV}$ ", CMS-PAS-SUS-16-042, cds.cern.ch/record/2257294

[5] CMS Collaboration, "Search for new physics in final states with two opposite-sign, same-flavor leptons, jets, and missing transverse momentum in pp collisions at $\sqrt{s}=13 \mathrm{TeV}$ ",

CMS-PAS-SUS-16-034, cds.cern.ch/record/2257293

[6] C. Lester and D. Summers, "Measuring masses of semiinvisibly decaying particles pair produced at hadron colliders", Phys. Lett. B 463 (1999) 99, doi:10.1016/S0370-2693(99)00945-4, arXiv:hep-ph/9906349

[7] CMS Collaboration, "Search for physics beyond the standard model in events with two leptons of same sign, missing transverse momentum, and jets in proton-proton collisions at $\sqrt{s}=13 \mathrm{TeV}$ ", Eur. Phys. J. C 77 (2017) 578, doi:10.1140/epjc/s10052-017-5079-z, arXiv:1704.07323

[8] CMS Collaboration, "Search for new physics with multileptons and jets in $35.9 \mathrm{fb}^{-1}$ of pp collision data at $\sqrt{s}=13 \mathrm{TeV} "$, CMS-PAS-SUS-16-041, cds.cern.ch/record/2256435 